\title{
Overview of Physics Results from MAST
}

H Meyer ${ }^{1}$, R J Akers ${ }^{1}$, F Alladio ${ }^{2}$, L C Appel ${ }^{1}$, K B Axon ${ }^{1}$, N Ben Ayed ${ }^{3}$, P Boerner ${ }^{11}$, R J Buttery ${ }^{1}$, P G Carolan ${ }^{1}$, D Ciric ${ }^{1}$, C D Challis ${ }^{1}$, I T Chapman ${ }^{1}$, G Coyler ${ }^{1}$, J W Connor ${ }^{1}$, N J Conway ${ }^{1}$, S Cowley ${ }^{1}$, M Cox ${ }^{1}$, G F Counsell ${ }^{1}$, G Cunningham ${ }^{1}$, A Darke ${ }^{1}, \mathbf{M}$ deBock $^{1}$, G deTemmerman ${ }^{1}$, R O Dendy ${ }^{1}$, J Dowling ${ }^{1}$, A Yu Dnestrovskij ${ }^{4}$, Yu N Dnestrovskij ${ }^{4}$, B Dudson ${ }^{3}$, D Dunai ${ }^{5}, \mathbf{M}$ Dunstan $^{1}$, A R Field ${ }^{1}$, A Foster ${ }^{6}$, L Garzotti $^{1}$, K Gibson ${ }^{3}$, M P Gryaznevich ${ }^{1}$, W Guttenfelder ${ }^{7}$, N C Hawkes ${ }^{1}$, J Harrison ${ }^{3}$, P Helander ${ }^{8}$, T C Hender ${ }^{1}$, B Hnat ${ }^{7}$, D F Howell ${ }^{1}$, M Duc Hua ${ }^{9}$, A Hubbard ${ }^{10}$, M Istenic ${ }^{1}$, N Joiner ${ }^{9}$ D Keeling ${ }^{1}$, A Kirk ${ }^{1}$, H R Koslowski ${ }^{11}$, Y Liang ${ }^{11}$, M Lilley ${ }^{9}$, S Lisgo ${ }^{1}$, B Lloyd ${ }^{1}$, G P Maddison ${ }^{1}$, R Maingi ${ }^{13}$, A Mancuso ${ }^{2}$, S J Manhood ${ }^{1}$, R Martin ${ }^{1}$, G J McArdle ${ }^{1}$, J McCone $^{14}$, C Michael ${ }^{1}$, P Micozzi ${ }^{2}$, T Morgan ${ }^{3}$, A W Morris ${ }^{1}$, D G Muir ${ }^{1}$, E Nardon ${ }^{1}$, G Naylor ${ }^{1}$, M R O'Brien ${ }^{1}$, T O'Gorman ${ }^{14}$, A Patel ${ }^{1}$, S D Pinches ${ }^{1}$, J Preinhaelter ${ }^{15}$, M N Price $^{1}$, E Rachlew ${ }^{12}$, D Reiter ${ }^{11}$, C M Roach ${ }^{1}$, V Rozhansky ${ }^{16}$, S Saarelma ${ }^{1}$, A Saveliev ${ }^{17}$, R Scannell ${ }^{1}$, S E Sharapov ${ }^{1}$, V Shevchenko ${ }^{1}$, S Shibaev ${ }^{1}$, H Smith ${ }^{9}$, G E Staebler ${ }^{18}$, D Stork ${ }^{1}$, J Storrs ${ }^{1}$, A Sykes ${ }^{1}$, S Tallents ${ }^{9}$, P Tamain ${ }^{1}$, D Taylor ${ }^{1}$, D Temple ${ }^{9}$, N ThomasDavies $^{1}$, A Thornton $^{3}$, A. Thyagaraja ${ }^{1}$, M R Turnyanskiy ${ }^{1}$, J Urban ${ }^{15}$, M Valovic ${ }^{1}$, R G L Vann ${ }^{3}$, F Volpe ${ }^{1}$, G Voss ${ }^{1}$, M J Walsh ${ }^{1}$, S E V Warder $^{1}$, R Watkins ${ }^{1}$, H R Wilson ${ }^{3}$, M Windridge $^{9}$, M Wisse ${ }^{1}$, A Zabolotski ${ }^{1}$, S Zoletnik ${ }^{4}$, O Zolotukhin ${ }^{1}$ and the MAST and NBI teams

${ }^{1}$ EURATOM/UKAEA Fusion Association, Culham Science Centre, Abingdon, UK.

${ }^{2}$ Associazione EURATOM-ENEA sulla Fusione, Frascati,Rome, Italy.

${ }^{3}$ University of York, Heslington, York, UK.

${ }^{4}$ Russian Research Centre,Kruchatov Institute, Institute of Nuclear Fusion, Moscow, Russia.

${ }^{5}$ KFKI-RMKI, Association EURATOM, Pf. 49, H-1525 Budapest, Hungary

${ }^{6}$ University of Strathclyde, Glasgow, UK.

${ }^{7}$ Department of Physics, Warwick University, UK.

${ }^{8}$ Max-Planck-Institute for Plasma Physics, Greifswald, Germany.

${ }^{9}$ Imperial College of Science, Technology and Medicine, London,UK.

${ }^{10}$ MIT Plasma Science and Fusion Centre, Cambridge, MA 02139, USA.

${ }^{11}$ Forschungszentrum Jülich GmbH, Association EURATOM-FZ Jülich, Institut für

Plasmaphysik, Trilateral Euregio Cluster, D-52425 Jülich, Germany

${ }^{12}$ Dept. of Physics, KTH, EURATOM -VR Association, Stockholm, Sweden.

${ }^{13}$ Oak Ridge National Laboratory, Oak Ridge, USA.

${ }^{14}$ University College, Cork, Association EURATOM-DCU Ireland.

${ }^{15}$ EURATOM/IPP.CR Fusion Association, Prague, Czech Republic.

${ }^{16}$ St. Petersburg State Polytechnical University, St. Petersburg, Russia.

${ }^{17}$ Ioffe Institute, Politekhnicheskaya 26, 194021 St. Petersburg, Russia.

${ }^{18}$ General Atomics, P.O. Box 85608, San Diego, California 92186-5608, USA.

E-mail: Hendrik.Meyer@ukaea.org.uk 
Abstract: Several improvements to the MAST plant and diagnostics have facilitated new studies advancing the physics basis for ITER and DEMO, as well as for future spherical tokamaks. Using the increased heating capabilities $P_{\mathrm{NBI}} \leq 3.8 \mathrm{MW} \mathrm{H}$-mode at $I_{\mathrm{p}}=1.2 \mathrm{MA}$ was accessed showing that the energy confinement on MAST scales more weakly with $I_{\mathrm{p}}$ and more strongly with $B_{\mathrm{t}}$ than in the ITER IPB98(y,2) scaling. Measurements of the fuel retention of shallow pellets extrapolate to an ITER particle throughput of $70 \%$ of its original design value. The anomalous momentum diffusion, $\chi_{\phi}$, is linked to the ion diffusion, $\chi_{i}$, with a Prandtl number close to $P_{\phi} \approx \chi_{\phi} / \chi_{i} \approx 1$, although $\chi_{i}$ approaches neoclassical values. New high spatially resolved measurements of the edge radial electric field, $E_{r}$, show that the position of steepest gradients in electron pressure and $E_{r}$ are coincident, but their magnitudes are not linked. The $T_{\mathrm{e}}$ pedestal width on MAST scales with the $\sqrt{\beta_{\text {pol }}}$ rather than $\rho_{\text {pol }}$. The ELM frequency for type-IV ELMs, new in MAST, was almost doubled using $n=2$ resonant magnetic perturbations from a set of 4 external coils $(n=1,2)$. A new internal 12 coil set $(n \leq 3)$ has been commissioned. The filaments in the inter-ELM and L-mode phase are different from ELM filaments, and the characteristics in L-mode agree well with turbulence calculations. A variety of fast particle driven instabilities were studied from $10 \mathrm{kHz}$ saturated fishbone like activity up to $3.8 \mathrm{MHz}$ compressional Alfvén eigenmodes (CAE). The damping rate of ellipticityinduced AE was measured to be $4 \%$ using the new internal coils as antennae. Fast particle instabilities also affect the off-axis NBI current drive and lead to fast ion diffusion of the order of $0.5 \mathrm{~m}^{2} / \mathrm{s}$ and reduce the driven current fraction from $40 \%$ to $30 \%$. EBW current drive start-up is demonstrated for the first time in a spherical tokamak generating plasma currents up to $55 \mathrm{kA}$. Many of these studies contributed to the physics basis of a planned upgrade to MAST.

1. Introduction: MAST [1] is one of the two leading tight aspect ratio $\left(A=\varepsilon^{-1}=R / a=\right.$ $\left.0.85 \mathrm{~m} / 0.65 \mathrm{~m} \sim 1.3, I_{\mathrm{p}} \leq 1.5 \mathrm{MA}\right)$ tokamaks in the world. The hot $T \leq 3 \mathrm{keV}$, dense $n_{\mathrm{e}}=(0.1-1) \times 10^{20} \mathrm{~m}^{-3}$ and highly shaped $(\delta \leq 0.5,1.6 \leq \kappa \leq 2.5)$ plasmas are accessed at moderate toroidal field $B_{\mathrm{t}}(R=0.7 \mathrm{~m}) \leq 0.62 \mathrm{~T}$ and show many similarities to conventional aspect ratio tokamaks. Detailed physics studies using the extensive array of state of the art diagnostics and access to different physics regimes help to consolidate the physics basis for ITER and DEMO [2,3], and explore the viability of future devices based on the spherical tokamak (ST) concept such as a component test facility (CTF) [4] or an advanced power plant [5]. The challenge for today's experiments is to find an integrated scenario that extrapolates to these future devices, in particular to develop plasmas with reduced power load on plasma facing components, notably from edge localised modes (ELM), but high confinement facilitated by internal or edge transport barriers. For steady state tokamak operation with tokamaks noninductive current drive techniques, including off-axis, have to be explored and high bootstrap current is needed. Fast particle instabilities, more prominent in future devices due to the $\alpha$-particle heating, affect the performance of these devices by broadening the fast particle distribution or spawning more detrimental instabilities. On MAST studies of ELM and pedestal characteristics (Sec. 3) and scrape-off layer transport (Sec. 4) help to consolidate our understanding of the plasma edge. These investigations are done with and without resonant magnetic perturbations for ELM mitigation (Sec. 3.2) or in the presence of pellet fuelling (Sec. 3.4). Core transport and confinement (Sec. 5) are scrutinised by detailed modelling as well as scaling experiments. With its high fraction of super Alfvénic particles, MAST is ideally suited to study the impact of fast particle driven instabilities (Sec. 6) on confinement, momentum transport (Sec. 5.3), off-axis current drive (Sec. 7.2), and develop general understanding of fast particle instabilities. More specific for the ST is the development of non-inductive start-up using 
electron Bernstein waves (EBW, Sec. 7.1). Many of the studies benefited from the enhanced plant and diagnostic capabilities (Sec. 2).

2. Diagnostic and Plant improvements: During the last two years MAST had major plant improvements. The neutral beam heating power was upgraded to $P_{\mathrm{NBI}} \leq 3.8 \mathrm{MW}$ by by replacing one Duopigatron source $\left(P_{\mathrm{NBI}} \leq 1.7 \mathrm{MW}, \Delta t \leq 0.4 \mathrm{~s}\right)$ with a JET style PINI injector $\left(P_{\mathrm{NBI}} \leq 2.5 \mathrm{MW}, \Delta t \leq 5 \mathrm{~s}\right)$. A similar upgrade is underway for the second beam line. A new toroidal arrays of 12 internal four turn coils ( 6 above, and 6 below the mid-plane every $60^{\circ}$ ) with 2 sets of power supplies enabling low current $I_{\mathrm{TAE}}^{\mathrm{AC}} \leq 4 \mathrm{~A}$ AC operation up-to $500 \mathrm{kHz}$ (TAE), and high current $I_{\mathrm{RMP}}^{\mathrm{DC}} \leq 1.4 \mathrm{kA}$ DC operation in each coil (RMP). These coils are used for TAE excitation (Sec. 6) and ELM mitigation (Sec. 3.2) studies respectively. The error field correction coils (EFC) have been equipped with new power supplies similar to the DC power supplies of the internal coils freeing 2 fast amplifiers $(f \leq 1 \mathrm{kHz}$ ) to improve the vertical feedback control. A $t \leq 90 \mathrm{~ms} P_{\mathrm{RF}} \leq 0.1 \mathrm{MW}$ gyrotron for start-up studies has also been commissioned (Sec. 7.1).

With respect to diagnostic enhancements the most notable are the new 35 channel MSE system [6] $\left(\Delta r \leq 2.5 \mathrm{~cm}, \Delta t \leq 5 \mathrm{~ms}\right.$, pitch angle resolution $\left.\Delta \alpha \leq 0.5^{\circ}\right)$, the upgrade of the NdYag based Thomson scattering (TS) system from a $200 \mathrm{~Hz} 4$ laser system with $E_{\text {las }} \approx 0.9 \mathrm{~J}$ to an 8 laser $240 \mathrm{~Hz}$ system with $E_{\text {las }} \approx 1.5 \mathrm{~J}$, the new 120 chord edge Doppler system, and the unique long wavelength IR camera $8.0 \mu \mathrm{m} \leq \lambda \leq 10.5 \mu \mathrm{m}$. The improvement of the Thomson scattering system is part of an ongoing upgrade, which will bring the spatial resolution of the NdYag system with 120 channels into the region of the ion Larmor radius $\rho_{i} \approx 1 \mathrm{~cm}$ with a factor of two larger signal. These diagnostic improvements are augmented by a recently developed interactive, integrated analysis package $\left(\mathrm{MC}^{3}\right)$ to improve the overall data consistency. A further major change is the upgrade of a large fraction of the data acquisition hardware to allow $5 \mathrm{~s}$ sampling at up to $10 \mathrm{MHz}$ sampling rate.

\section{H-mode edge, ELM stability and fuelling:}

3.1. ELMs and pedestal: Using the better NdYag edge TS system on the low field side (LFS) [7] in conjunction with the high field side (HFS) data from the Ruby system earlier studies on the comparison of LFS and HFS pedestals with the Ruby system alone $[8,9]$ were revisited. The study covered a wide data set with $0.5 \mathrm{MA} \leq I_{\mathrm{p}} \leq 1.2 \mathrm{MA}, 0.2 \leq n_{\mathrm{e}} / n_{\mathrm{G}} \leq 0.9\left(n_{\mathrm{G}}\right.$ : Greenwald density), $3 \leq q_{95} \leq 7,0.45 \mathrm{~T} \leq B_{\mathrm{t}} \leq 0.55 \mathrm{~T}$, triangularity of $0.3 \leq \delta \leq 0.5$, elongation of $1.6 \leq \kappa \leq 2.1$ and auxiliary heating power of $1.0 \mathrm{MW} \leq P_{\mathrm{NBI}} \leq 3.8 \mathrm{MW}$ in double null (DN) and single null (SN) configurations [10].

Of particular interest is the different behaviour of the density pedestal width, $\Delta_{n_{\mathrm{e}}}$, in DN and SN. In DN $\Delta_{\mathrm{n}_{\mathrm{e}}}$ is narrower in flux space on the HFS than on the LFS, but not so in SN. In contrast, the temperature, $\Delta_{T_{\mathrm{e}}}$, and interestingly also the pressure, $\Delta_{p_{\mathrm{e}}}$, pedestal widths are similar in flux space on the HFS and LFS in both configurations. This has two major consequences in DN. Firstly, the electron pressure is not a flux function, and secondly, the HFS location for the $n_{\mathrm{e}}$ barrier and $T_{\mathrm{e}}$ barrier are not the same. In DN $\Delta_{n_{\mathrm{e}}}$ is the same in real space on the HFS and the LFS showing the importance of the ionisation source for the density pedestal formation. This may be explained by the fact that the scrape-off-layer (SOL) on the HFS is much narrower in DN than in SN [11]. 


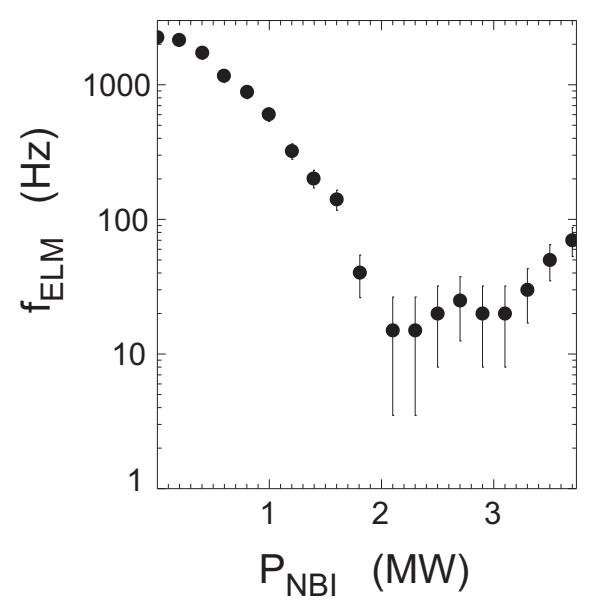

Figure 1: Variation of ELM repetition rate with NBI power.

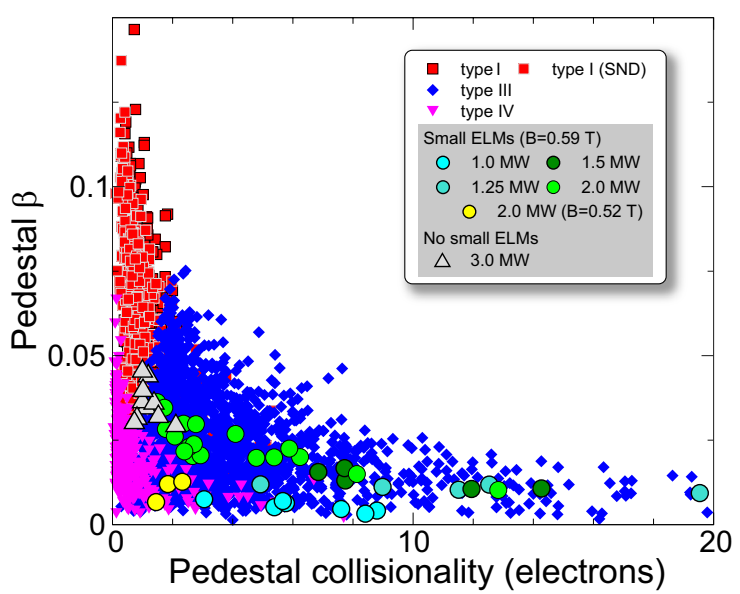

Figure 2: Existence space of different ELM types with respect to electron $\beta$ and $v^{\star}$ at the top of the pedestal.

Three different large ELM types can be distinguished in this data set: ELMs at high edge collisionality $\left(T_{\mathrm{e}}^{\text {ped }} \lesssim 0.15 \mathrm{keV}, n_{\mathrm{e}}^{\text {ped }} \gtrsim 2 \times 10^{19} \mathrm{~m}^{-3}\right)$ where the repetition rate decreases with increasing power (type-III); ELMs at lower edge collisionality $\left(T_{\mathrm{e}}^{\text {ped }} \gtrsim 150 \mathrm{eV}, n_{\mathrm{e}}^{\text {ped }} \gtrsim\right.$ $2.5 \times 10^{19} \mathrm{~m}^{-3}$ ), but high density where the repetition rate slowly increases with power (type-I, Fig. 1); ELMs at low collisionality and low density $\left(T_{\mathrm{e}}^{\mathrm{ped}} \gtrsim 100 \mathrm{eV}, n_{\mathrm{e}}^{\text {ped }} \lesssim 2.5 \times 10^{19} \mathrm{~m}^{-3}\right)$ with characteristics of type-IV ELMs (or the low collisionality branch of type-III ELMs). In addition a type of small ELMs with $n \sim 30$ co-current rotating filaments and distinct regular mode structure has been observed. Only a few of these filaments detach. At high pedestal pressure approaching the type-I ELM regime, the small ELMs vanish (Fig. 2). A comparison to small ELMs in NSTX and Alcator C-MOD [12] shows that these ELMs are distinct from the small type-V ELMs observed on NSTX [13], because of the different mode structure and rotation direction.

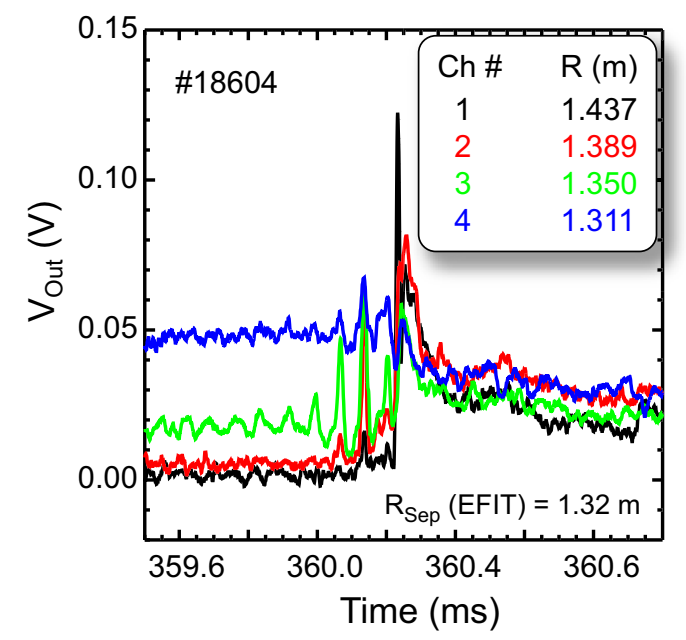

Figure 3: BES signal at the plasma edge during a type-I ELM.
In SN only type-I ELMs have been observed [10], although the edge stability is not changed by the configuration [9]. These type-I ELMs are sometimes preceded by a precursor as can be seen from Fig. 3 showing data from a trial 8 channel beam emission spectroscopy (BES) system. This burst of edge localised fluctuations is locked in frequency to a steady core $n=1$ MHD mode (Sec. 6). For typeI ELMs the ideal edge stability analysis performed is consistent with the peeling-ballooning theory for ELM triggering $[14,15]$. The profiles just prior to a type-I ELM are close to the peeling-ballooning stability boundary, whereas those for the type-III and type-IV ELMs are in the ideal MHD stable region and are assumed to be driven by resistive modes.

The type-IV ELM regime as well as the data from different magnetic configurations help to shed light on the pedestal width scaling with respect to the co-linearity between the normalised 
poloidal Larmor radius $\rho_{\mathrm{pol}}^{\star}$ and $\beta_{\mathrm{pol}}$. From the picture of turbulence suppression by $E \times B$ flow shear one would expect the pressure pedestal width to change like $\Delta_{p} / a=2 \sqrt{6 \varepsilon /(1+\varepsilon)} \rho_{\text {pol }}^{\star}$. On MAST, however, very little change of $\Delta_{T_{e}}$ is observed with respect to $\rho_{\text {pol }}^{\star}$, but $\Delta_{T_{\mathrm{e}}}$ clearly increases with $\beta_{\text {pol }}$ in suitable comparison discharges. This is similar to the recent findings of $\Delta_{p} / a \propto\left(\rho_{\mathrm{pol}}^{\star}\right)^{0.1} \beta_{\mathrm{pol}}^{0.5}$ on JT60-U using deuterium and hydrogen discharges [16]. From the full data set one gets a scaling $\Delta_{T_{\mathrm{e}}} / a \propto \beta_{\mathrm{pol}}^{\alpha}$ with $\alpha_{\mathrm{DN}}=0.52 \pm 0.03$ and $\alpha_{\mathrm{SN}}=0.47 \pm 0.05$ in DN and $\mathrm{SN}$ respectively neglecting the $\rho_{\text {pol }}^{\star}$ dependence.

3.2. ELM mitigation: For studies of ELM mitigation by resonant magnetic perturbations MAST is now equipped with two coil sets: The new internal (RMP) coil set (Sec. 2) similar to the DIII-D I-coils [17], and the external error field correction coil set (EFC) with four mid-plane three turn coils ( $I_{\mathrm{EFC}} \leq 5 \mathrm{kA}$ each). This set-up gives flexibility in the toroidal mode spectrum $n \leq 3$ and phasing, with the poloidal spectrum determined by the pitch angle and the current parity of the upper and lower internal coils [18].

With an $n=1$ perturbation from the EFC coil set similar to the recent JET studies [19] locked modes were triggered before ELMs were affected, although the locked mode threshold [20] on MAST should be higher, and the Chirikov parameter $\sigma_{\text {Chirikov }}[21,22]$ is higher indicating more edge ergodisation (Fig. 4). In the $n=2$ configuration an increase of the ELM frequency from the natural frequency of $f_{\mathrm{ELM}} \approx 0.6 \mathrm{kHz}$ to $f_{\mathrm{ELM}}^{\mathrm{EFC}} \approx 1 \mathrm{kHz}$ has been observed in the low collisionality type IV ELM regime. Here, only one of the two coil pairs was connected to give an $n=2$ perturbation and the other was used to cancel partly the intrinsic

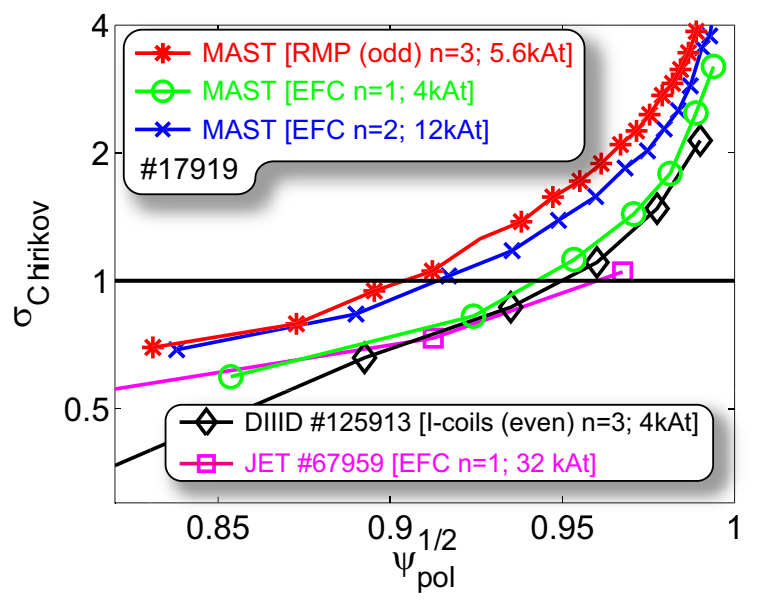

Figure 4: Chirikov parameter as function of normalised radius for DIII-D $\diamond$, JET $\square$, $M A S T \times(E F C n=2), \circ(E F C n=1), \star$ $(R M P n=3)$.

error field. Commissioning studies with the new RMP coil set in Ohmic discharges show a distinct density pump-out during the period when the q-profile is resonant to the perturbation. In this period there seems also to be a small change towards more positive $E_{r}$ as expected for an ergodised edge.

3.3. Radial electric field: The evolution and structure of the radial electric field, $E_{r}$, on MAST in L-mode and H-mode has been studied using edge Doppler spectroscopy [23]. $E_{r}$ is derived from the radial force balance of the observed ion species (here $\mathrm{He}^{+}$at $\lambda_{0}=468.6 \mathrm{~nm}$ ). Using a local gas puff a spatial resolution of the $E_{r}$ profile of $\Delta r=(3-6) \mathrm{mm}$ is achieved with a typical time resolution of $\Delta t=5 \mathrm{~ms}$ on roughly 60 spatial points with a chord distance of $1.5 \mathrm{~mm}$. The evolution of $E_{r}$ through an $\mathrm{L}$ to $\mathrm{H}$ transition is shown in Fig. 5. In $\mathrm{H}$-mode a narrow negative edge well evolves in $E_{r}$ with a typical minimum of $E_{r}^{\min } \approx-15 \mathrm{kV} / \mathrm{m}$.

In Fig. 6 the electron pressure gradient versus the electric field gradient calculated over the whole pedestal region is shown for two different $\mathrm{H}$-mode discharges well into an ELM free phase. These discharges with different plasma current, $I_{\mathrm{p}}=0.7 \mathrm{MA}$ and $I_{\mathrm{p}}=1.1 \mathrm{MA}$, and 


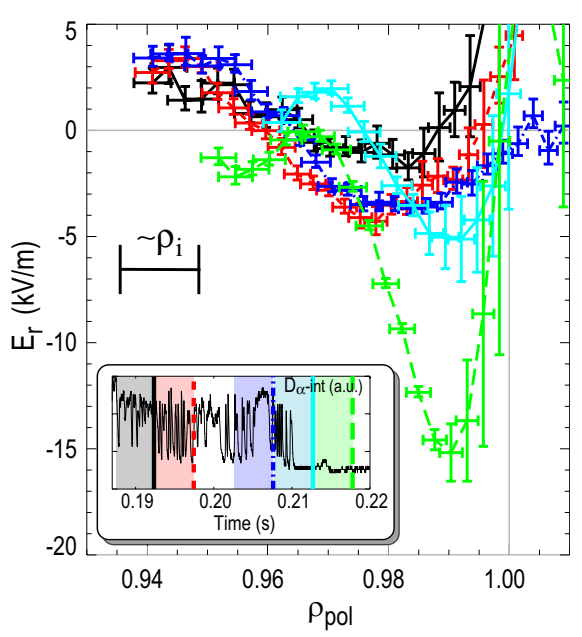

Figure 5: Evolution of the radial electric field through an $L-H$ transition on MAST.

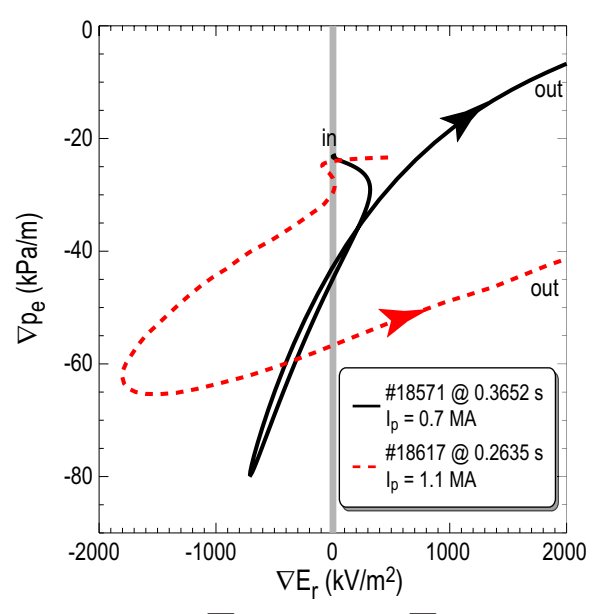

Figure 6: $\nabla p_{\mathrm{e}}$ versus $\nabla E_{r}$ in the pedestal region for a low (black, solid) and high (red, dashed) current $H$-mode (arrows indicate $R$ ). different fuelling were chosen because of their distinct difference in the width of the $E_{r}$ well and the pedestal. The high current discharge has a much narrower $E_{r}$ well, but a shallower pedestal pressure compared to the low current discharge. For both discharges clearly the strongest negative pressure gradient, $\nabla p_{\mathrm{e}}$ is associated with the negative gradient in $E_{r}$. The magnitude of $\nabla p_{\mathrm{e}}$ is not correlated with $\nabla E_{r}$ suggesting that other physical processes limit the $\nabla p_{\mathrm{e}}[23]$.

3.4. Pellet fuelling: Using an 8 barrel pellet injector the particle transport in MAST was studied with pneumatically accelerated pellets. Typical pellet speeds between $250 \mathrm{~m} / \mathrm{s} \leq$ $v_{\text {pellet }} \leq 400 \mathrm{~m} / \mathrm{s}$ from the high field side (HFS) top of the machine result in shallow pellet injection between $0.6 \leq \rho_{\text {pel }}=\sqrt{\psi_{\mathrm{N} \text {,pel }}} \leq 0.9$ The pellet trajectory has been observed using unfiltered high speed visible imaging as well as time averaged narrow band filtered visible imaging in a line free region at $\lambda=(523.44 \pm 0.32) \mathrm{nm}$ to measure bremsstrahlung. These data are used to establish the pellet deposition radius $\rho_{\text {pel }}$ [24]. High resolution TS measurements with the single time Ruby system event triggered from the pellet system itself are used to determine the pellet retention time $\tau_{\text {pel }}[24,25]$.

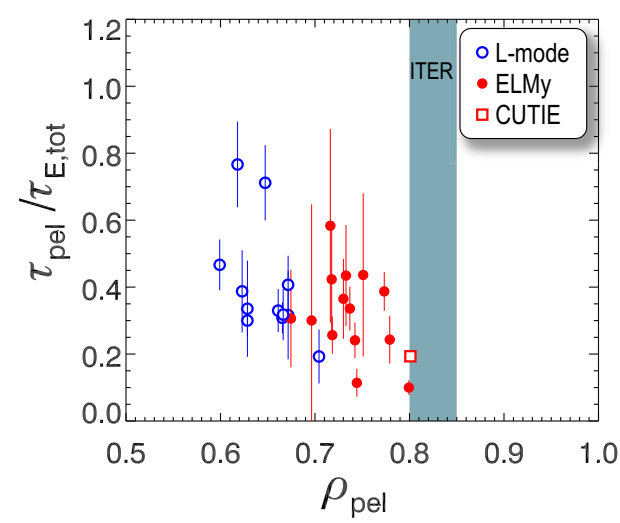

Figure 7: Pellet retention time normalised to $\tau_{\mathrm{E}}$ as function of pellet deposition radius for L-mode (blue open circles) and ELMy H-mode (red full circles).
Fig. 7 shows that at ITER like deposition radius the retention time normalised to the energy confinement time in ELMy H-mode is only $\tau_{\mathrm{pel}} / \tau_{\mathrm{E}}=0.2$. Hence the particle throughput estimated for ITER $\Phi_{\text {pel }} \approx n_{\mathrm{e}} S(a-$ $\left.r_{\mathrm{pel}}\right) / \tau_{\mathrm{pel}} \approx 70 \mathrm{Pam}^{3} / \mathrm{s}$ is about $70 \%$ of the originally foreseen ITER design value. Here nominal values are used for ITER [2] of $n_{\mathrm{e}}=10^{20} \mathrm{~m}^{-3}, S=683 \mathrm{~m}^{2}$ and $\tau_{\mathrm{E}}=3.7 \mathrm{~s}$. Comparisons with predictive pellet ablation codes show that the $\nabla B$-drift as well as the plasma precooling due to the drift is needed in order to understand the post pellet $n_{\mathrm{e}}$ profiles on MAST. The mostly adiabatic response of the kinetic profiles to the perturbation by the pellet leads to a shorter $T_{\mathrm{e}}$ gradient length $L_{T_{\mathrm{e}}}=T_{\mathrm{e}} /\left(\nabla T_{\mathrm{e}}\right)$ in the region of the positive density gradient. This leads to an increased turbulence level in this region according to calculations with the GS2 [26] and CUTIE [27] codes. This increased turbulence leads to 
an increased inwards particle flux which is needed to understand the pellet retention time [24]. As can be seen from the square in Fig. 7 the pellet retention time simulated with CUTIE by approximating the pellet particle source is in good agreement with the experiment.
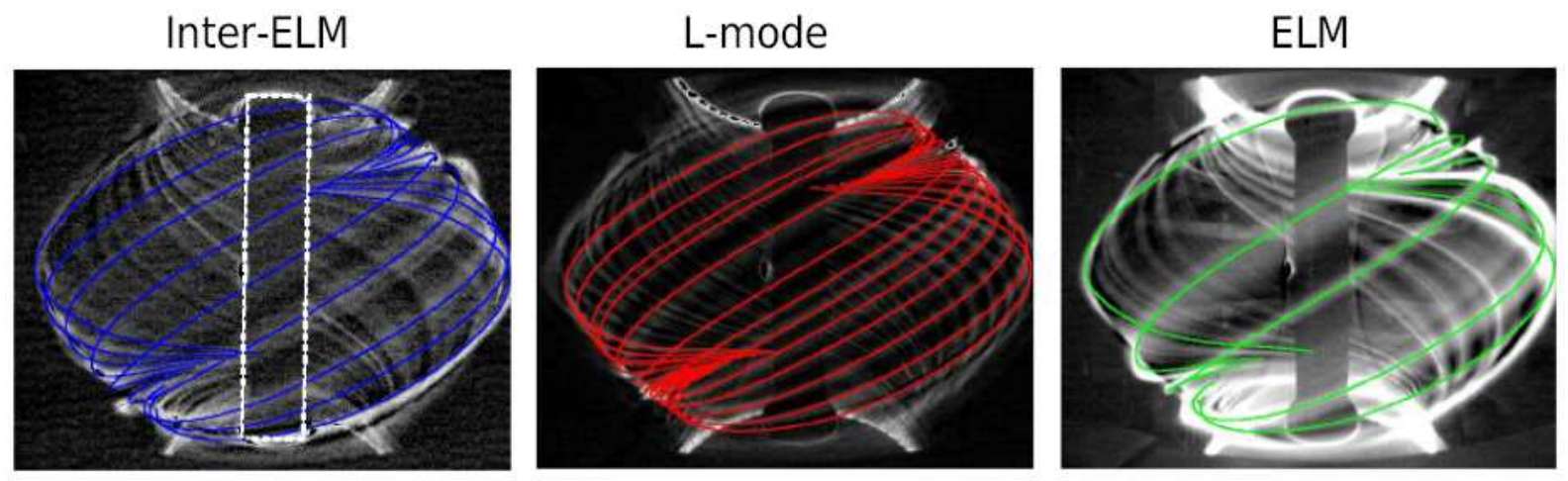

Figure 8: Filamentary structures in the inter ELM period (left), L-mode (middle) and during an ELM (right) with the magnetic field lines overlayed.

4. SOL modelling and Edge filaments: In recent years much attention was devoted to ELM filaments [28-30] (Fig. 8 right), but it has been long known that the intermittent L-mode transport is also filamentary [31,32] (Fig. 8 middle). Careful background subtraction shows that these field-aligned filaments are also present in inter ELM periods [33] (Fig. 8 left). The characteristics of these filaments as deduced from the visible imaging are listed in Table 1 [34]. This comparison suggests that the sporadic inter-ELM filaments are more closely related to L-

Table 1: Filament properties from fast imaging and BOUT L-mode modelling ( $\tau$ : life time, $v_{r, \phi}$ : radial, toroidal velocity, $N$ number of filaments, $L_{\mathrm{rad}, \theta}:$ radial, poloidal scale length)

\begin{tabular}{ccccccc}
\hline Regime & $\tau(\mu \mathrm{s})$ & $v_{r}(\mathrm{~km} / \mathrm{s})$ & $v_{\phi}(\mathrm{km} / \mathrm{s})$ & $N$ & $L_{\mathrm{rad}}(\mathrm{cm})$ & $L_{\theta}(\mathrm{cm})$ \\
\hline L-mode & $40-60$ & $0.5-1.5$ & $2-9$ & $20-50$ & $5-10$ & $7-9$ \\
Inter-ELM & $50-120$ & $1-2$ & $3-12.5$ & $10-40$ & $3-5$ & $9-12$ \\
ELM & $100-180$ & $1 \rightarrow 9$ & $10-30$ & $10-20$ & $4-6$ & $2-6$ \\
\hline BOUT L-mode & & $\sim 0.5$ & & $\sim 40$ \\
\hline
\end{tabular}

mode filaments than to ELM filaments (e.g. drift waves rather than ideal MHD). The L-mode turbulence was modelled using the BOUT code [35] in good agreement with the experimental data. It should be noted that the small ELMs described in Sec. 3.1 are different from the interELM filaments with respect to their mode structure. In contrast to the inter-ELM filaments, the small ELM shows a very regular sharp mode structure in the visible imaging. Empirical modelling to include filamentary effects on SOL transport is underway [36].

5. Core Confinement and Transport: Transport in MAST is investigated using analytic theory and a variety of codes including gyro-kinetic (flux-tube: GS2; global: ORB5, GYRO) and mesoscale MHD turbulence simulations, predictive modelling with reduced theoretical models, and interpretative modelling [37]. In many cases, the experimentally observed toroidal flow shear is sufficient to suppress long wavelength (ITG) turbulence. Predictive modelling of 
non sawtoothing L-mode discharges was done with GLF23, TGLF, and ORB5 [37, 38]. GLF23 and TGLF tend to underestimate the core transport $(r / a<0.4)$ with $E \times B$ shear stabilisation enabled (in particular for the electrons) and overestimate the ion transport without $E \times B$ shear. ORB5 without $E \times B$ shear underestimates core transport. TGLF predicts electron transport dominated by high-k (ETG) turbulence, but other mechanisms such as MHD or fast particle driven micro-tearing may also cause significant electron transport leading to the less peaked $T_{\mathrm{e}}$ and $T_{\mathrm{i}}$ profiles as observed and predicted by CUTIE (albeit for circular flux surfaces and zero particle trapping). The non linear CPTM model [39] has been used on H-mode discharges, giving reasonable agreement for $n_{\mathrm{e}}$ and $T_{\mathrm{e}}$ profiles.

5.1. Energy confinement scaling: The MAST H-mode confinement scaling data base was expanded to higher plasma current $I_{\mathrm{p}} \leq 1.2 \mathrm{MA}$ and higher heating powers $P_{\mathrm{NBI}} \leq 3.8 \mathrm{MW}$. Furthermore, dedicated scaling experiments with respect to the toroidal field $B_{\mathrm{t}}$ and $I_{\mathrm{p}}$ have been performed. The data now approach the $I_{\mathrm{p}} / I_{\text {rod }} \approx 0.65$ of the ST-CTF design point [4] at stored energies of $W_{\text {mag }}=150 \mathrm{~kJ}$. With respect to dimensionless parameters the MAST data set connects to CTF values in $\rho_{\star}, q_{\text {eng }}$, and $\beta_{\mathrm{N}}$ but extrapolation with respect to $v_{\star}$ is still more than an order of magnitude. In terms of engineering parameters the MAST data set as well as the dedicated scans support a slightly weaker scaling of $\tau_{\mathrm{E}}$, with $I_{\mathrm{p}}$ and a much stronger scaling with $B_{\mathrm{t}}$ than the IPB98(y,2) scaling [25]. This is in agreement with results from NSTX [40]. Depending on the scaling method the coefficients for $I_{\mathrm{p}}$ and $B_{\mathrm{t}}$ are $0.4 \leq \alpha_{I_{\mathrm{p}}} \leq 0.6$ and $1.3 \leq \alpha_{B_{\mathrm{t}}} \leq 1.6$ respectively compared to $\alpha_{I_{\mathrm{p}}}=0.93$ and $\alpha_{B_{\mathrm{t}}}=0.15$ for ITP98(y,2). Such a scaling extrapolates to $H_{\mathrm{H}}=\tau_{\mathrm{E}} / \tau_{\text {scal. }} \approx 1.6$ for the current ST-CTF design point compared the assumed $H_{\mathrm{H}}=1.3$. Transport analysis indicates that the different scaling of $\tau_{\mathrm{E}}$ with $I_{\mathrm{p}}$ and $B_{\mathrm{t}}$ in ST's may arise from a dependence of the effective heat diffusivity of $\chi_{\text {eff }} \propto \chi_{\mathrm{GB}} q^{\alpha_{q}} v_{\star}^{\alpha_{v}}$ with $\alpha_{q} \lesssim 1$ and $\alpha_{v} \approx 3 / 4$ ( $\chi_{\text {GB }}$ gyro-Bohm diffusivity). This is slightly weaker in $q$ and stronger in $\nu_{\star}$ than found in conventional tokamaks.

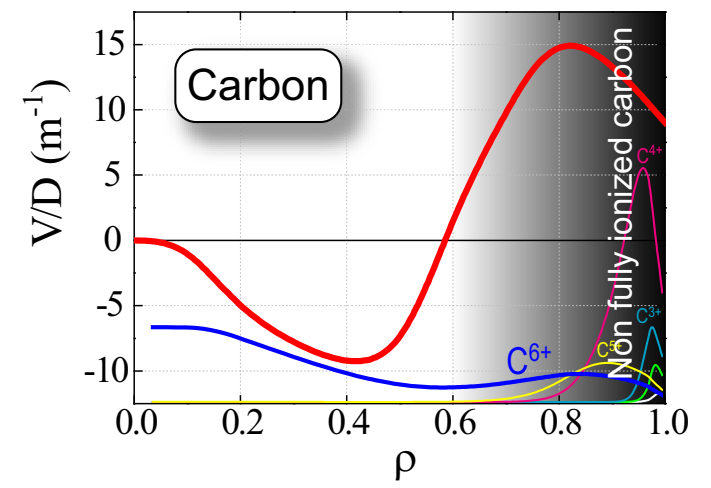

Figure 9: Modelled ratio of impurity pinch velocity and impurity diffusion coefficient for a MAST L-mode discharge using the STRAHL [41] code (also shown: profiles of ionisation states).
5.2. Particle and impurity confinement: A number of quasi steady state L-mode discharges was used to investigate the global particle transport in MAST by looking at the parameter dependence of the density peaking $\hat{n}=n_{\mathrm{e}, 0} /\left\langle n_{\mathrm{e}}\right\rangle$. A strong inverse correlation of $\gamma_{j_{\star} \hat{n}}=-0.62$ is seen with the dimensionless, averaged current density $j_{\star}=$ $\mu_{0} I_{\mathrm{p}} R /\left(S B_{\mathrm{t}}\right)$ on MAST $\left(\gamma_{x y}=\left(\sum x_{i} y_{i}-n \bar{x} \bar{y}\right) /[(n-\right.$ 1) $\left.\sigma_{x} \sigma_{y}\right] ; \sigma$ the standard deviation). A slightly stronger correlation of $\gamma_{\hat{n} \bar{s}}=0.72$ is observed with $\bar{s}=q_{95} / q_{0}$, and a weak negative correlation with loop voltage $V_{\text {loop. }}$. Almost no correlation is seen with $v_{\star}$. In the $\hat{n}$ versus $j_{\star}$ space the MAST data are well aligned with TCV data from Ref. [42]. The strong correlation with $j_{\star}$ and not $V_{\text {loop }}$ suggests the presence of a turbulent pinch [43] with the Ware pinch playing a minor role.

A pinch in the core $(r / a<0.6)$ of the order of $V_{Z} \approx-10 \mathrm{~m} / \mathrm{s}$ is also observed for the 
main impurity Carbon. In Fig. 9 the profile of the ratio of pinch velocity and diffusion $V_{Z} / D_{Z}$ for $\mathrm{C}^{6+}$ in a typical L-mode discharge is shown. The transport coefficient profiles are derived from modelling the measured $Z_{\text {eff }}$ profile by solving the particle balance $\partial n_{Z} / \partial t=$ $-\nabla\left\{-D_{Z} \nabla n_{Z}+V_{Z} n_{Z}\right\}+\Sigma_{j} S_{Z j}\left(S_{Z, j}\right.$ : source terms) using the STRAHL code [41] with a constant diffusion coefficient of $D_{Z}=1 \mathrm{~m}^{2} / \mathrm{s}$ consistent with a particle confinement time of $\tau_{p} \approx a^{2} /\left(6 D_{Z}\right) \approx 0.05 \mathrm{~s}$. Here, $\mathrm{C}$ is assumed to be the only impurity and the profiles of $T_{\mathrm{e}}$ and $n_{\mathrm{e}}$, as well as the $\mathrm{C}$ influx are measured. The modelling suggests an outward convection for the impurities of order of $V_{Z} \approx 15 \mathrm{~m} / \mathrm{s}$ in the periphery due to a minimum in the $Z_{\text {eff }}$ and $\mathrm{C}^{6+}$ profiles around $r / a \sim 0.6$. Such a minimum requires the reversal of $V_{Z}$ for realistic profiles of $D_{Z}$. The low impurity peaking in the core indicates that turbulent and neoclassical transport in the core are of the same order.

5.3. Momentum confinement: Understanding the plasma flow has become increasingly important in recent years. This is not only because of the impact on plasma confinement due to turbulence suppression by sheared flows [44,45], which give rise to internal transport barriers (ITB), but also due to the impact of flow on MHD stability [46,47]. Plasmas in NBI heated spherical tokamaks show a fast toroidal rotation with thermal Mach numbers measured on MAST of up to $M_{\mathrm{th}}=v_{\phi} / v_{\mathrm{th}} \lesssim 0.8$ [48]. On many tokamaks a link between momentum confinement and energy confinement has been observed [49], with the so called Prandtl number $P_{\phi}=\chi_{\phi} / \chi_{i} \approx 1$ relating ion momentum and ion energy diffusivity. This is consistent with theoretical studies of ITG transport. In particular in discharges with counter current NBI a substantial improvement of the confinement with plasma rotation is observed [50]. This can be readily understood by flow shear stabilisation of turbulence [44, 51], the shear on MAST being sufficient to stabilise ITG turbulence [51] (see above).

On MAST $P_{\phi}$ is also close to unity, as can be seen from Fig. 10 [52] showing the profile of $P_{\phi}$ for various time slices in a number of $\mathrm{L}$ mode (green) and H-mode (magenta) discharges calculated using the TRANSP code [53]. The data naturally have a large scatter, but clump around $P_{\phi} \approx 1$ for $0.1<\rho<0.7$ decreasing towards the edge. Even though $\chi_{i}$ approaches neoclassical values, momentum transport is still dominated by turbulent processes, because for neoclassical transport $\chi_{\phi}^{\text {neo }}<<\chi_{i}^{\text {neo }}$ (by one or two orders of magnitude). Hence, the residual turbulence contributes little to $\chi_{i}$ but dominates $\chi_{\phi}$. A

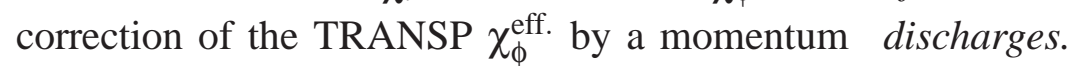

pinch generated by the "Coriolis drift" influencing small scale instabilities [54] $\chi_{\phi}=\chi_{\phi}^{\text {eff }}[1-$ $\left.\left(L_{\omega} V_{\phi}^{\text {pinch }}\right) / L_{n}\right]^{-1}$ with $R V_{\phi}^{\text {pinch }}=-\chi_{\phi}\left(R / L_{n}+4\right) / 2$ and $L_{n}$ the density gradient length, increases $P_{\phi}$ in the plasma edge, where $L_{n}$ is short, leading to $P_{\phi}>>1$.

6. Fast particle instabilities: The low toroidal field $B_{\mathrm{t}} \approx 0.5 \mathrm{~T}$ in beam heated STs has the consequence that the speed of the injected particles is well above the Alfvén velocity 
$v_{A}=B_{\mathrm{t}} / \sqrt{\left(\mu_{0} \sum_{i} n_{i} m_{i}\right)} \approx 1 \times 10^{6} \mathrm{~m} / \mathrm{s}$ for beam energies $E_{\mathrm{NBI}}>30 \mathrm{keV}$. Therefore, a wide variety of Alfvénic and fast particle driven activity is observed on MAST [55,56].

In particular a fast particle driven $n=1$ internal kink is observed with several harmonics at frequencies $f_{\mathrm{LLM}}=(10-80) \mathrm{kHz}$ [55]. The mode develops, as q evolves, out of $n=1$ chirping fishbone activity. In some shots the mode couples to low frequency tearing activity in other shots it has been observed to transform back into chirping fishbone activity. During the life time of this mode a reduction of core electron and ion temperature, core rotation and core density in $\mathrm{H}$-mode is observed. This can be interpreted by an increased loss of fast ions due to this mode, which is consistent with bolometer measurements sensitive to fast particle losses and TRANSP analysis showing a too high neutron rate.

Magnetic activity has been observed up to $f \leq 3.8 \mathrm{MHz}$. Modes with $f_{\mathrm{CAE}}=(0.6-3.8) \mathrm{MHz}$ have been identified as Compressional Alfvén Eigenmodes(CAE) by their elliptic polarisation with $\delta B_{\|}$of the order of $\delta B_{\perp}[55,57]$. An eigenmode analysis for the measured frequencies and mode numbers, together with the resonance condition for the beam, places these modes around mid radius on the LFS. In this region $2 \pi f_{\mathrm{CAE}} \gtrsim \omega_{\mathrm{ci}}$. CAE's have also been identified at lower frequencies around $\omega_{\mathrm{ci}} / 2$ suggesting that CAE's exist in two distinct ranges of $k_{\|}$. At lower frequency $f_{\mathrm{TAE}}=(0.1-0.2) \mathrm{MHz}$ the polarisation of toroidal Alfvén eigenmodes (TAE) has been measured showing their shear-Alfvén character.

6.1. TAE damping: Apart from studying the unstable modes as discussed in the previous section one can also study the stable modes by actively exciting them with an antenna. For this each of the 6 lower coils of the new internal 12 coils set (Sec. 3.2) can be connected to individual $500 \mathrm{~W}$ power amplifiers driving a maximum current $I_{\mathrm{int}}^{\mathrm{AC}} \leq 4 \mathrm{~A}$ with frequencies up to $f_{\text {coil }} \leq 0.5 \mathrm{MHz}$. The 6 upper coils are then used as detection coils supplementing the extensive set of LFS Mirnov coils.

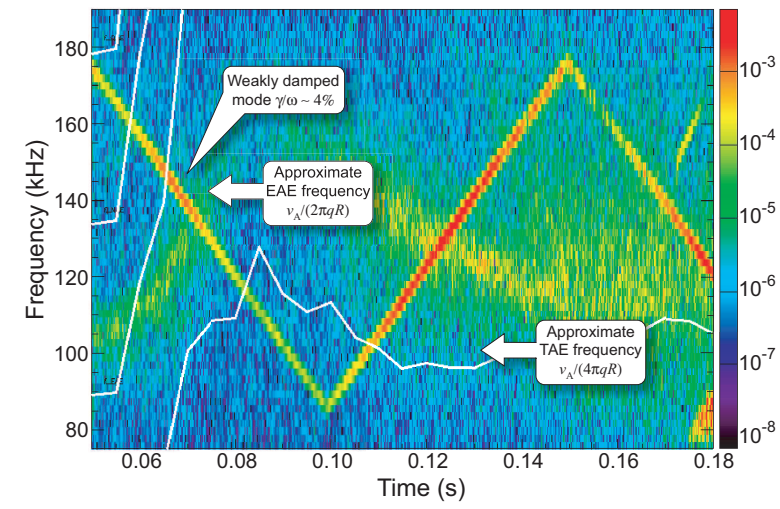

Figure 11: Frequency spectrum of LFS Mirnov coil during a TAE antenna sweep. stronger resonance overlapping modes preven
Initial experiments were started in 2007 using two of three test coils (toroidal locations: $0^{\circ}$, $60^{\circ}$, and $180^{\circ}$ ) in $n=1$ configuration. As can be seen from Fig. 11 showing the magnetic spectrogram during an antenna sweep from 85 $\mathrm{kHz}$ to $180 \mathrm{kHz}$, there are two resonances at $t_{1} \approx 65 \mathrm{~ms}$ and $t_{2} \approx 120 \mathrm{~ms}$ at frequencies $f_{E A E} \approx 0.14 \mathrm{MHz}$ the frequency of Ellipticityinduced Alfvén Eigenmodes [58] on MAST. At the first resonance damping rates of $\gamma / \omega \approx$ $-4 \%$ are measured, whereas at the second 7. Current Drive and Start-up: One of the major challenges for future steady state STs with high neutron flux is plasma start-up, current ramp-up and current sustainment without a high flux solenoid. Furthermore, low order rational surfaces have to be avoided by operating with $q_{\min }>1.3$, because of the detrimental impact of low $n$ MHD such as infernal modes [46], NTMs and "monster" sawteeth [47]. 
7.1. EBW start-up: A short pulse $(\Delta t \leq 90 \mathrm{~ms}) 100 \mathrm{~kW} f_{\mathrm{ECRH}}=28 \mathrm{GHz}$ ECRH system was commissioned to study plasma start-up with EBW current drive [59]. The waves are launched in O-mode $\left(n_{\text {cut-off }} \approx 10^{19} \mathrm{~m}^{-3}\right.$ ) from the LFS and converted to X-mode using a grooved tile at the mid-plane of the centre column as a mirror-polariser. At the upper hybrid resonance the X-mode waves are converted into electrostatic EBW waves [60] and subsequently absorbed at the Doppler shifted electron cyclotron resonance. The waves are launched with a poloidal angle of $\alpha \approx 10^{\circ}$ from below the mid-plane and are absorbed above the mid-plane. With a symmetrical vertical field, $B_{v}$ following the RF break-down a pressure driven current appears near the mid-plane slowly shifting downwards as a negative EBW driven current appears above the mid-plane. These two currents repel each other and lead to a decay of the total plasma current regardless of further $B_{v}$ ramp-up or RF injection. This loss of plasma current can be prevented by either shifting the plasma initially up until closed flux surfaces (CFS) form or by applying a concave curvature to the vertically field using the divertor coils with inverted current during the initial phase of the plasma formation. Once CFS are established the plasma is shifted to the vertically stable mid-plane position or the divertor coils can be operated in their normal polarisation. Using these two techniques hot plasmas with $n_{\mathrm{e}} \leq 2 \times 10^{18} \mathrm{~m}^{-3}$ and $T_{\mathrm{e}} \leq 0.7 \mathrm{keV}$ have been sustained for $200 \mathrm{~ms}$. The pure EBW driven current is $I_{\mathrm{p}}=17 \mathrm{kA}$ at constant $B_{v}$. With $B_{v}$ ramp-up this can be increased to $I_{\mathrm{p}}=33 \mathrm{kA}$, and by using less than $0.5 \%$ of the solenoid flux $\left(\Psi_{\text {sol }}=0.2 \mathrm{~V} \times 20 \mathrm{~ms}\right) I_{\mathrm{p}}=55 \mathrm{kA}$ is achieved.

7.2. Off-axis NBCD and current ramp-up optimisation: For future ST devices a broad current profile with $q_{\min }>1.3$ is needed to achieve high elongation with high bootstrap current and to avoid detrimental low $n$ MHD. In order to sustain such a profile one needs off-axis current drive, since the current diffusion as well as the on axis NBI will lead to a peaked current profile in the flat-top eventually. Off-axis NBI on MAST is achieved by shifting the magnetic axis of the plasma far off the mid-plane $\left(\Delta Z_{\mathrm{mag}} \lesssim 0.35 \mathrm{~m}\right)[61]$. $T_{\mathrm{e}}$, plasma energy $W_{\mathrm{pl}}$, and neutron rate $S_{n}$ in these extreme $\mathrm{SN}$ discharges $\left(I_{\mathrm{p}}=0.6 \mathrm{MA}, P_{\mathrm{NBI}}=3.5 \mathrm{MW}\right)$ are comparable to similar DN discharges, although the confinement of the beam is better in DN. This suggests a similar heating efficiency for both off-axis NBI and on-axis NBI in MAST.

Analysis using TRANSP suggests that about $30 \%$ of the total current is driven off-axis with the peak at $r / a \approx 0.4$ (Fig. 12). To match the measured neutron rate, $S_{n}$, and $W_{\mathrm{pl}}$ with TRANSP an ad-hoc anomalous fast ion diffusion of $D_{\mathrm{b}} \approx 0.5 \mathrm{~m}^{2} / \mathrm{s}$ was introduced. However, the discrepancy only exists from $t_{1}=0.2 \mathrm{~s}$ to $t_{2}=0.4 \mathrm{~s}$, otherwise $D_{\mathrm{b}} \approx 0$. During this time strong $n=1$ fishbone activity is observed. This activity may well lead to the radial transport of fast ions [62]. The effect of the anomalous fast ion diffusion on the current profile is shown in Fig. 12. The level is comparable to values reported from DIII-D [62] and ASDEX Upgrade [63].

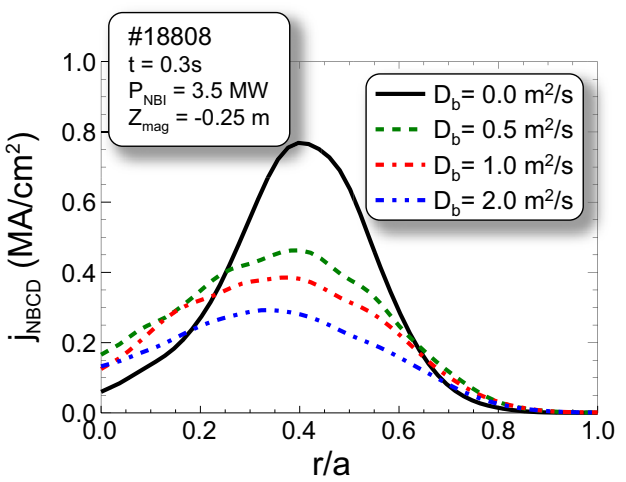

Figure 12: TRANSP simulations of $N B$ driven current for various values of anomalous fast particle diffusion $D_{\mathrm{b}}$ due to fishbones.

In future devices non inductive current ramp up, e.g. EBWCD and NBCD will, be used to form 
the current profile for the steady state. The high neoclassical resistivity in an ST, however, leads to a fast current penetration, and therefore to a rapid peaking of the current profile. This may be avoided by optimising the current ramp up with respect to heating, fuelling and current ramp rate. The q-profile formation during the ramp phase was analysed using TRANSP for a set of discharges with varying density and current ramp rates, as well as different NBI $\left(P_{\mathrm{NBI}}=1.4 \mathrm{MW}\right)$ onset during the current ramp $[64,65]$. The TRANSP runs were benchmarked against the $q_{\min }$ evolution estimated from the onset of Alfvén cascades [55] as well as the onset of other MHD. The q-profiles at the end of the current ramp were compared. Without NBI the density ramp rate has little effect on the always monotonic q-profile. With a faster current ramp the whole q-profile is elevated. Early NBI leads to a reversal of the q-profile due to off-axis $(r / a \approx 0.4-0.5)$ pile-up of Ohmic current (the NB driven current is only 5\%-7\%). This is more pronounced with the faster density ramp. The on axis NBI heats the core reducing the resistivity, therefore increasing the current penetration time. This heating is proportional to $I_{\mathrm{p}}$ and $n_{\mathrm{e}}$. Using this technique q-profiles with $q_{\mathrm{min}}>2$ were formed approaching those foreseen in the upgraded MAST.

8. Conclusions: Over the last two years research on MAST has made valuable contributions to a variety of areas important not only for future spherical tokamaks (ST), but importantly also for ITER and DEMO. This is facilitated by continuous improvements to plant, diagnostics and analysis techniques. Notable contributions to ITER and DEMO physics are in the areas of pellet fuelling, ELM and pedestal physics, as well as fast particle driven instabilities. More ST specific areas include off-axis neutral beam current drive (NBCD), and electron Bernstein wave current drive (EBWCD) start-up. Many of the studies done are original to the ST. In particular shallow pellet fuelling, ELM mitigation with off mid-plane $n=3$ coils, the active excitation of otherwise stable Alfvén eigenmodes, off-axis NBCD, and EBWCD start-up are unique in the ST. Here, a possible design limitation in the original ITER design with respect to particle throughput was revealed. The frequency of ELMs was increased with $n=2$ resonant magnetic perturbations using external coils. $30 \%$ off-axis current was driven with $P_{\mathrm{NBI}}=3.5 \mathrm{MW}$, and plasmas start-up with EBWCD was demonstrated $\left(I_{\mathrm{p}}=55 \mathrm{kA}\right.$ with $0.5 \%$ solenoid swing and $\left.P_{\mathrm{RF}}=0.1 \mathrm{MW}\right)$. In order to model MAST plasmas adequately turbulence, predictive transport and MHD codes are continuously improved by the requirements to include strong flow shear, finite pressure $\beta$ (electromagnetic effects), and finite Larmor radius physics. For example the new TGLF reduced transport model is benchmarked using MAST discharges giving reasonable agreement if $E \times B$ flow shear is included. The different energy confinement scaling for the ST with weaker $I_{\mathrm{p}}^{0.6}$ and stronger $B_{\mathrm{t}}^{1.3}$ dependence than the IPB98 $(\mathrm{y}, 2)$ scaling may already emphasise the differences expected in future high $\beta$ regimes and could lead to a different design optimisation for an STCTF. The near isotropic fast particle distribution at the the resonant Alfvén velocity mimics burning plasmas and Alfvńic activity with elliptic polarisation is observed with frequencies up to $f=3.8 \mathrm{MHz}$ of the order of the ion cyclotron frequency. The fast particle driven activity is already seen to affect neutral beam heating, current drive and torque. Challenging measurements like the structure of the edge radial electric field with resolution better than the ion Larmor radius, the q-profile (motional Stark effect) at low magnetic field, or the local density fluctuations (beam emission spectroscopy) allow new physics to be investigated such as 
the interplay between pressure gradient and electric field gradient. The characterisation of the dynamic of inter-ELM and L-mode filaments will guide the interpretation of scrape-off layer transport. The research also consolidates the physics basis of the planned upgrade to MAST, designed to study current drive and fast particle physics, as well as divertor power handling and pumping in long pulses with relaxed current profile.

Acknowledgements: This work was jointly funded by the UK Engineering and Physical Sciences Research Council and by the European communities under the Contract of Association between EURATOM and UKAEA. The views and opinions expressed herein do not necessarily reflect those of the European Commission. NBI equipment is on loan from ORNL, the NPA from PPPL and the pellet injector was provided by FOM. Plasma control software was provided by GA.

\section{References}

[1] DARKE, A. et al., MAST: A mega amp spherical tokamak, in Proceedings of the 18th Symposium on Fusion Technology, 1994.

[2] ITER-TEAM, ITER Physics Basis, volume 39, chapter 2, pages 2175-2249, Nucl. Fusion, 1999.

[3] IKEDA, K. et al., Nucl. Fusion 47 (2007) S1.

[4] VOSS, G. et al., Fusion Eng. and Design (2008), in press.

[5] WILSON, H. et al., Nucl. Fusion 44 (2004) 917.

[6] KULDKEPP, M. et al., Rev. Sci. Instr. 77 (2006) 10E905.

[7] SCANNELL, R. et al., Rev. Sci. Instr. 77 (2006) 10E510.

[8] KIRK, A. et al., Plasma Phys. Control. Fusion 46 (2004) A187.

[9] MEYER, H. et al., Nucl. Fusion 46 (2006) 64.

[10] KIRK, A. et al., H-mode pedestal characteristics on MAST, submitted to Plasma Phys. Contr. Fusion.

[11] MEYER, H. et al., Plasma Phys. Control. Fusion 47 (2005) 843.

[12] MAINGI, R. et al., Comparison of small ELM characteristics and regimes in Alcator C-MOD, MAST, and NSTX, in Proc. 22nd IAEA FEC, 2008.

[13] MAINGI, R. et al., Nucl. Fusion 45 (2005) 1066.

[14] WILSON, H. R. et al., Phys. of Plasmas 9 (2002) 1277.

[15] SNYDER, P. B. et al., Pedestal Stability Comparison and ITER Pedestal Prediction, in Proc. 22nd IAEA FEC, 2008.

[16] URANO, H. et al., Nucl. Fusion 48 (2008) 045008 (9pp).

[17] EVANS, T. et al., Nature Physics 2 (2006) 419.

[18] KIRK, A. et al., ELM power loadings and control on MAST using resonant magnetic perturbations, in Proc. 22nd IAEA FEC, 2008.

[19] LIANG, Y. et al., Phys. Rev. Lett. 98 (2007) 265004.

[20] HOWELL, D. F. et al., Nucl. Fusion 47 (2007) 1336.

[21] CHIRIKOV, B. V., Journal of Nuclear Energy. Part C, Plasma Physics, Accelerators, Thermonuclear Research $1(1960) 253$.

[22] NARDON, E. et al., Journ. Nucl. Mat. (2008), to be published, preprint available.

[23] MEYER, H. et al., Journ. Phys.: Conf. Series 123 (2008) 012005.

[24] VALOVIC, M. et al., Nucl. Fusion 48 (2008) 075006 (8pp).

[25] VALOVIC, M. et al., Confinement and fuelling in MAST, in Proc. 22nd IAEA FEC, 2008.

[26] KOTSCHENREUTHER, M. et al., Comp. Phys. Comm. 88 (1995) 128.

[27] ThYagarajA, A. et al., Phys. of Plasmas 12 (2005) 090907.

[28] KIRK, A. et al., Phys. Rev. Lett. 92 (2004) 245002.

[29] KIRK, A. et al., Plasma Phys. Control. Fusion 46 (2004) 551.

[30] ZOHM, H., Plasma Phys. Control. Fusion 38 (1996) 105.

[31] GOODALL, D., Journ. Nucl. Mat. 111 (1982) 11.

[32] ZWEBEN, S. J. et al., Nucl. Fusion 23 (1983) 825. 
[33] BEN AYED, N. et al., Experimental observation of inter-ELM filaments on MAST, in Proc. 35th EPS Plasma Physics Conference, 2008, to be published in Plasm. Phys. Contr. Fusion.

[34] DUDSON, B. et al., Experiments and simulation of the edge turbulence and filaments in MAST, in Proc. 35th EPS Plasma Physics Conference, 2008, submitted to Plasm. Phys. Contr. Fusion.

[35] XU, X. Q. et al., Nucl. Fusion 40 (2000) 731.

[36] LISGO, S. et al., The Influence of Filaments on Scrape-Off Layer Transport, in Proc. 22nd IAEA FEC, 2008.

[37] AKERS, R. et al., Transport studies in the MAST spherical tokamak, in Proc. 22nd IAEA FEC, 2008.

[38] STAEBLER, G. M. et al., Testing the Trapped Gyro-Landau Fluid Transport Model with Data from Tokamaks and Spherical Tori, in Proc. 22nd IAEA FEC, 2008.

[39] DNESTROVSKIJ, A. et al., Canonical Profiles Transport Model for H-mode shots in Tokamaks, in Proc. 22nd IAEA FEC, 2008.

[40] KAYE, S. et al., Nucl. Fusion 46 (2006) 848.

[41] BEHRINGER, K., R (87) 8 (1987).

[42] ZABOlOTSKY, A. et al., Plasma Phys. Control. Fusion 45 (2003) 735.

[43] GARBET, X. et al., Phys. Rev. Lett. 91 (2003) 035001.

[44] TERRY, P. W., Rev. Mod. Phys. 72 (2000) 109.

[45] WOLF, R. C., Plasma Phys. Control. Fusion 45 (2003) R1.

[46] MENARD, J. E. et al., Nucl. Fusion 43 (2003) 330.

[47] CHAPMAN, I. T. et al., Plasma Phys. Control. Fusion 49 (2007) B385.

[48] AKERS, R. J. et al., Plasma Phys. Control. Fusion 45 (2003) A175.

[49] TALA, T. et al., Plasma Phys. Control. Fusion 49 (2007) B291.

[50] COUNSELL, G. et al., Nucl. Fusion 45 (2005) S157.

[51] FIELD, A. et al., Core Heat Transport in the MAST Spherical Tokamak, in Proc. 20th IAEA Fusion Energy Conf. on Fusion Energy 2004 (Vilamoura, 2004), 2004.

[52] FIELD, A. R. et al., Momentum transport in MAST spherical tokamak plasmas, in Proc. 35th EPS Plasma Physics Conference, 2008, to be published in Plasm. Phys. Contr. Fusion.

[53] HAWRYLUK, R. J., An emperical approach to tokamak transport, in Physics of plasmas close to thermonuclear conditions, edited by COPPI, B. et al., volume 1, CEC, Brussels, 1980.

[54] PEETERS, A. G. et al., Phys. Rev. Lett. 98 (2007) 265003.

[55] GRYAZNEVICH, M. et al., Nucl. Fusion 48 (2008) 084003.

[56] PINCHES, S. et al., Fast particle instabilities in MAST, in Proc. 22nd IAEA FEC, 2008.

[57] APPEL, L. C. et al., Plasma Phys. Control. Fusion (2008), accepted.

[58] BETTI, R. et al., Physics of Fluids B: Plasma Physics 4 (1992) 1465.

[59] SHEVCHENKO, V. et al., Phys. Rev. Lett. 89 (2002) 265005.

[60] LAQUA, H. P., Plasma Phys. Control. Fusion 49 (2007) R1.

[61] LLOYD, B. et al., Nucl. Fusion 47 (2007) S658.

[62] HEIDBRINK, W. W. et al., Nucl. Fusion 42 (2002) 972.

[63] GÜNTER, S. et al., Nucl. Fusion 44 (2005) S98.

[64] KEELING, D. et al., Tayloring the q-profile on MAST for scenario optimisation, in Proc. 35th EPS Plasma Physics Conference, 2008.

[65] TURNYANSKIY, M. R. et al., Current profile control studies on MAST, in Proc. 22nd IAEA FEC, 2008. 